\title{
Diseño de un fluido de perforación que disminuya el impacto ambiental de los suelos en el proceso de disposición final
}

\author{
ANDREA MARISELA CHAMORRO GARCÍA ${ }^{*}$ * EDIXON JOSÉ COLMENARES GUTIÉRREZ², \\ ODGUIL ANTONIO HARNISTH PINOS! \\ 1 Universidad Central del Ecuador, Facultad de Ciencias Químicas, Gatto Sobral y Leiton, Quito. \\ 2 Weatherford, Avenida Amazonas 1014 y Naciones Unidas, Edif. La Previsora, Quito. \\ *correspondencia: andreinaquimica@hotmail.com
}

Recibido: 25 de febrero de 2015, Aceptado: 10 de agosto de 2016

\section{Resumen}

Los procesos de disposición final de fluidos de perforación en la industria petrolera, se ven influenciados por la forma en que los contaminantes se desechan y luego se disuelven en el suelo. El objetivo de esta investigación consiste en formular un fluido de perforación base agua con inhibidores químicos, que presenten las propiedades reológicas apropiadas, que su disposición final sea menos nociva para el suelo y que actúen como fertilizantes, en dicho fluido de perforación se utilizaron inhibidores químicos como: sulfato de potasio, nitrato de potasio y formiato de sodio a diferentes concentraciones. Una vez cumplido los 180 días de disposición del fluido en el suelo se evaluó el pH y la conductividad eléctrica, en base a la ley hidrocarburífera, Decreto 1215. Los resultados demostraron que la formulación del fluido de perforación que contiene almidón $8.69 \mathrm{~g} / \mathrm{L}$, carboximetil-celulosa 2,89g/L, goma xantana $1,44 \mathrm{~g} / \mathrm{L}$, hexadiamina $83,3 \mathrm{~mL} / \mathrm{L}$, carbonato de calcio $246,16 \mathrm{~g} / \mathrm{L}$, bentonita $57 \mathrm{~g} / \mathrm{L}$ y sulfato de potasio $34,75 \mathrm{~g} / \mathrm{L}$, fue la más adecuada, evidenciándose con el desarrollo de una planta de fréjol que creció, aproximadamente, catorce centímetros en siete días. Es importante recalcar que el uso de sales inorgánicas como inhibidores químicos en los fluidos de perforación no afecta las propiedades reológicas y su disposición final disminuye la contaminación del suelo.

Palabras clave: contaminación de suelo, fertilizante, fluido de perforación, inhibidor de arcilla, reología.

\section{Design of a drilling fluid to decrease the environmental impact Of soils in the process of disposal.}

\section{Abstract}

The processes of final disposal of drilling fluids in the oil industry are influenced by the way the pollutants are disposed and then dissolved in soil. The aim of this research consisted of formulating a water based drilling fluid with chemical inhibitors that present the proper rheological properties, so that their final disposal becomes less harmful to the soil and can act as fertilizer. Such chemicals as potassium sulfate, potassium nitrate, and sodium formate, were used in different concentrations. Upon completion of the 180 days of the fluids' disposal in soil, $\mathrm{pH}$ and electrical conductivity were evaluated according to the Hydrocarbon Law, Decree 1215. The results showed that the drilling fluid, which contains starch $8.69 \mathrm{~g} / \mathrm{L}$, carboxymethylcellulose $2.89 \mathrm{~g} / \mathrm{L}$, xanthan gum $1.44 \mathrm{~g} / \mathrm{L}$, hexadiamine $83.3 \mathrm{~mL} / \mathrm{L}$, calcium carbonate $246.16 \mathrm{~g} / \mathrm{L}$, bentonite 57 $\mathrm{g} / \mathrm{L}$, and potassium sulfate $34.75 \mathrm{~g} / \mathrm{L}$, was the most suitable. This fact was evidenced by the development of a bean plant that grew approximately fourteen centimeters in seven days. It is important to emphasize that the use of inorganic salts as chemical inhibitors in drilling fluids does not affect the rheological properties and their final disposal decreases pollution of soils.

Keywords: soil pollution, fertilizer, drilling fluid, clay inhibitor, rheology. 


\section{Introducción}

Tomando en cuenta que el petróleo es la mayor fuente de energía que mueve al mundo, se hace cada vez más imprescindible el optimizar su extracción y uso pues el desarrollo económico en el planeta ha hecho que uno de los productos de mayor demanda sea el petróleo. Iniciada la década de los setenta se produce una ampliación y diversificación en la industria petrolera, caracterizada por altos precios del petróleo en el mercado mundial, permitiendo el crecimiento económico del Ecuador.

El Ecuador en sus 40 años, aproximadamente, de explotación petrolera, mantiene un historial bastante nefasto para la economía nacional en cuanto a la degradación de los ecosistemas naturales. Un elemento que genera inconformidad y conflictos entre comunidades y compañías petroleras, es el incumplimiento de la Ley Hidrocarburífera, dentro de las fases de exploración, explotación, industrialización, comercialización y abandono de una actividad petrolera. La empresa debe tomar en cuenta algunas normas de comportamiento ambiental necesarias para desarrollar una gestión sustentable de los recursos.

En la perforación de un pozo de petróleo es vital el uso de un fluido de perforación, ya que éste cumple funciones importantes dentro del proceso. En Ecuador, se perfora con fluidos base aceite y agua, los fluidos en base agua son mejores debido a su menor costo e impacto ambiental en relación a otros tipos de fluidos de perforación.

De los fluidos de perforación depende el éxito o fracaso de la producción de hidrocarburos. Durante su disposición final se genera contaminación del suelo, pues con el tiempo el fluido de perforación se convierte en un desecho provocando un impacto ambiental y debe ser eliminado de conformidad con los reglamentos ambientales locales como la Ley Hidrocarburífera (Decreto 1215).

La explotación petrolera en el norte de la Amazonía ecuatoriana es responsable de la deforestación de dos millones de hectáreas debido principalmente al derrame del crudo y disposición final de los fluidos de perforación con formulaciones base aceite y ciertas sustancias tóxicas como los cloruros (Ministerio del Ambiente, 2012).
El impacto ambiental más grande de la historia de la explotación petrolera, fue en la provincia de Orellana, causado por la empresa transnacional Texaco, el $60 \%$ es suelo contaminado por la incorrecta disposición de líquidos y desechos industriales, fluidos de perforación, y residuos sólidos, provocando daños a la flora, la fauna y en sí al hombre, ya que dichas actividades afectaron a varias etnias indígenas, como los Cofanes, Sionas y Secoyas, hasta estar en peligro de desaparición (SENPLADES, 2009).

El término "fluido de perforación", incluye: gas, aire, petróleo, agua, y suspensiones coloidales a base de agua y arcilla, que circula a través de la sarta de perforación hasta la barrena y regresa a la superficie, de igual forma poseen propiedades físicas y químicas que le permiten adaptarse a una gran variedad de condiciones. El fluido de perforación es una parte clave del proceso de perforación y producción de petróleo y/o gas, y el éxito depende de su diseño.

\section{Inhibidores químicos}

La utilización de inhibidores como el $\mathrm{K}_{2} \mathrm{SO}_{4^{\prime}} \mathrm{KNO}_{3}$ y $\mathrm{NaCOOH}$ se debe, principalmente, a la interacción química de la arcilla que presenta con dichos compuestos, función de fertilización en suelos, control de impacto ambiental, y su economía. En la actualidad se utilizan las sales de cloruro pero estas son altamente reguladas, por razones ambientales comprobadas químicamente en el campo petrolero.

La interacción química de la arcilla depende del catión, ya que puede servir de enlace para mantener unidas a las partículas del mineral arcilloso, el ion potasio sirve como catión de intercambio ya que tiene un diámetro muy pequeño y como consecuencia calza perfectamente en los huecos hexagonales de la capa de silicio. De esta manera, las capas sucesivas están unidas fuertemente y hace que la perforación sea adecuada utilizando dicho fluido con características modificadas químicamente.

El tamaño y la forma del catión afectan su capacidad de encajar entre las superficies entre capas de la arcilla y afectan tanto a la viscosidad del fluido como a la hidratación de la arcilla. Los espacios dentro de las 
capas de la arcilla que se forman son de $2,8 \AA$, los pequeños iones como el potasio, que encajan entre ellas son intercambiados con mayor facilidad y de manera permanente, estimulando la hidratación de la arcilla permitiendo que el fluido de perforación esté en mejores condiciones para el trabajo de pozos (Tabla 1).

Tabla 1. Tamaño de los cationes

\begin{tabular}{cc}
\hline Catión & Diámetro (Å) \\
\hline Na- Sodio & 1.90 \\
K- Potasio & $\mathbf{2 . 6 6}$ \\
Cs- Cesio & 3.34 \\
Mg- Magnesio & 1.30 \\
Ca- Calcio & 1.90 \\
\hline
\end{tabular}

Adaptado de (Instituto Americano del Petróleo, 2001)

\section{Sulfato de potasio}

El sulfato de potasio $\left(\mathrm{K}_{2} \mathrm{SO}_{4}\right)$ es la sal de potasio del ácido sulfúrico. También denominado arcanita, y se utiliza como una alternativa directa del cloruro de potasio.

Entre las aplicaciones del sulfato de potasio está su utilización para formular fluidos de perforación a base de agua donde se requiere una fuente de iones de potasio para inhibir la hidratación de lutitas y se prohíben las sales de cloruro por la contaminación que ocasiona. El sulfato de potasio se utiliza exclusivamente en operaciones de descarga en tierra.

Cuando el sulfato de potasio se introduce en el fluido, el magnesio y calcio solubles se precipitan, al igual que sus sulfatos. Por consiguiente, se recomienda controlar el $\mathrm{pH}$ del fluido antes de agregar el sulfato de potasio.

\section{Ventajas}

- Proporciona una alternativa comprobada para el cloruro de potasio.

- Permite el uso de potasio en áreas donde el uso y la descarga del cloruro están regulados.

\section{Nitrato de potasio}

El nitrato de potasio $\left(\mathrm{KNO}_{3}\right)$ es un componente del salitre, aproximadamente el $75 \%$ del nitrato de potasio se manufactura con una pureza del $90 \%$ para su uso como fertilizante.

El anión nitrato $\left(\mathrm{NO}_{3}\right)$, adicionado como nitrato de potasio, es la fuente más usada como fertilizante por su fácil pérdida de degradación en la disposición final de fluidos de perforación.

En su utilización hay que tener precaución ya que por su composición y características, cuando se aporta este inhibidor en suelos con alto porcentaje de saturación de agua baja sensiblemente su $\mathrm{pH}$ y su aportación de nitrógeno en forma nítrica, puede ocasionar riesgos ambientales.

\section{Ventaja}

- Permite fácil degradación en el suelo.

\section{Formiato de sodio}

Los formiatos son una clase de sal que proviene del ácido fórmico, las salmueras de formiato no contienen sólidos, no hay agentes densificantes que aumenten los volúmenes de desechos contaminados.

El uso de salmueras de formiato puede disminuir la carga tóxica total en el ambiente que la recibe, tienen un alto potencial para recuperación y re-uso, reduciendo de este modo el volumen total de descargas químicas, presenta a los operadores oportunidades de minimizar el impacto de su disposición final, reduciendo el volumen de desperdicio total y toxicidad al eliminar los agentes densificantes y minimizar los aditivos químicos y materiales densificantes sólidos en el desperdicio. 


\section{Ventajas}

- Las salmueras de formiato son densificados naturalmente.

- Estabiliza los polímeros con facilidad.

\section{Descarga o disposición final de}

\section{desechos al ambiente}

Es depositar o confinar permanentemente residuos en sitios e instalaciones cuyas características permitan prevenir su liberación al ambiente y las consecuentes afectaciones a la salud de la población y a los ecosistemas y sus elementos (Petroproducción, 1992).

El nivel de impacto que una descarga puede tener sobre el medio ambiente depende de los tipos de materiales contenidos en los desechos y del ambiente donde son descargados.

El diseño de este fluido de perforación tiene la finalidad de contener compuestos químicos que no afecten sus propiedades reológicas para el proceso de perforación y que en su disposición final el suelo pueda ser reutilizado sin necesidad de biorremediación.

\section{Parte experimental}

\section{Materiales y equipos}

- Potenciómetro

- Balanza analítica Mettler Toledo, modelo AL204, apreciación $\pm 0.1 \mathrm{mg}$, rango de pesaje; máximo $210.0 \mathrm{~g}$, mínimo $0.01 \mathrm{~g}$

- Viscosímetro rotativo / fann Model 207198 (35A)

- Conductímetro

\section{Reactivos de grado técnico}

- Agua de pozo lajustar pH para la formulación)

- Hidróxido de sodio

- Almidón
- Carboximetil celulosa

- Goma xantana

- Carbonato de calcio

- Hexadiamina

- Sulfato de potasio

- Nitrato de potasio

- Formiato de sodio

- Bentonita

\section{Procedimiento}

- Se elaboró el fluido de perforación en el laboratorio utilizando los criterios expuestos en el manual de fluidos de perforación del Instituto Americano de Petróleos (API), registrando densidad, viscosidad plástica, viscosidad aparente y punto cedente, parámetros de control que se evalúan constantemente en los fluidos antes de ser sometidos al proceso de perforación.

- Se coloca un litro de agua, y se ajusta el pH entre 9-9.5 con $\mathrm{NaOH}$, se calienta a una temperatura de $100^{\circ} \mathrm{C}$ y se añade almidón y goma xantana, con agitación constante evitando que se formen grumos, se espera que la mezcla esté completamente homogénea, se adiciona cuidadosamente la carboximetilcelulosa, dejando que se enfríe la mezcla sin suspender la agitación y se coloca la mezcla en un agitador "Hamilton Beach" y se adiciona la hexadiamina, bentonita y carbonato de calcio, se añade los inhibidores sulfato de potasio, nitrato de potasio o formiato de sodio en las concentraciones respectivas.

- Se evalúan los parámetros de control del fluido de perforación de acuerdo a la Ley hidrocarburífera actual (Decreto 1215).

- Acceso y descarga a los sitios donde se realizará la disposición final del fluido de perforación. 
(Existen lugares específicos en donde se realiza la descarga de estos fluidos de perforación, como referencia a estos se ocupó una parcela de tierra en el Oriente ecuatoriano que cumple con las mismas características de las parcelas de tierra reales que actualmente se utilizan).

- Se toma las muestras de suelo seleccionadas en las parcelas del campo Palo Azul. (A los 170 días se sembró fréjol AFROANDINO en las parcelas, como sistema de control)

- Se recolecta las muestras de suelo en fundas de plástico (Fernandez, Rojas, Roldán, \& Ramirez, 2006).

- Se lleva las muestras hacia las instalaciones del laboratorio ambiental de la empresa.

- Se analiza los parámetros físicos-químicos, $\mathrm{pH}$ y conductividad eléctrica.

- Se realiza un tratamiento estadístico de los datos obtenidos para establecer los resultados.

\section{Resultados y discusión}

\subsection{Resultados}

El objetivo de este proyecto fue diseñar un fluido de perforación, amigable al ambiente en su disposición final utilizando sales orgánicas e inorgánicas en una formulación base agua expuesta por una entidad experta en fluidos de perforación (Tabla 2).

Tabla 2. Formulación del fluido de perforación original. $I_{0}-C_{0}$

\begin{tabular}{cc}
\hline Producto & Concentración (g/L) \\
\hline Almidón & 8.69 \\
Carboximetil-Celulosa & 2.89 \\
Goma Xantana & 1.44 \\
Hexadiamina & $83.33 \mathrm{ml} / \mathrm{L}$ \\
Carbonato de Calcio & 246.16 \\
Bentonita & 57 \\
\hline
\end{tabular}

Las siguientes tablas (Tabla 3 a 5) indican la proporción de sales orgánicas e inorgánicas que se agregaron como inhibidores químicos al fluido de perforación, según la fórmula base con la que se trabajó.

Tabla 3. Fluido de perforación con inhibidor sulfato de potasio

\begin{tabular}{|l|c|c|c|c|}
\hline & Base 1 & \multicolumn{2}{c|}{ Base 2 } & \multicolumn{2}{c|}{ Base 3 } & Base 4 \\
& $\mathrm{I}_{1}-\mathrm{C}_{1}$ & $\mathrm{I}_{1}-\mathrm{C}_{2}$ & $\mathrm{I}_{1}-\mathrm{C}_{3}$ & $\mathrm{I}_{1}-\mathrm{C}_{4}$ \\
\hline \multicolumn{1}{|c|}{ Producto } & & \multicolumn{2}{c|}{ Concentración, $\mathbf{~ m g / L}$} \\
\hline Almidón & 8.69 & 8.69 & 8.69 & 8.69 \\
\hline Carboximetil-Celulosa & 2.89 & 2.89 & 2.89 & 2.89 \\
\hline Goma Xantana & 1.44 & 1.44 & 1.44 & 1.44 \\
\hline Hexadiamina & $83.33 \mathrm{~mL} / \mathrm{L}$ & $83.33 \mathrm{~mL} / \mathrm{L}$ & $83.33 \mathrm{~mL} / \mathrm{L}$ & $83.33 \mathrm{~mL} / \mathrm{L}$ \\
\hline Carbonato de Calcio & 246.16 & 246.16 & 246.16 & 246.16 \\
\hline Bentonita & 57 & 57 & 57 & 57 \\
\hline Sulfato de Potasio & 11.58 & 23.16 & 34.75 & 46.33 \\
\hline
\end{tabular}


Tabla 4. Fluido de perforación con inhibidor nitrato de potasio

\begin{tabular}{|c|c|c|c|c|}
\hline & Base 5 & Base 6 & Base 7 & Base 8 \\
& $\mathrm{I}_{2}-\mathrm{C}_{1}$ & $\mathrm{I}_{2}-\mathrm{C}_{2}$ & $\mathrm{I}_{2}-\mathrm{C}_{3}$ & $\mathrm{C}_{4}$ \\
\hline Producto & \multicolumn{4}{|c|}{ Concentración, mg/L } \\
\hline Almidón & 8.69 & 8.69 & 8.69 & 8.69 \\
\hline Carboximetil-Celulosa & 2.89 & 2.89 & 2.89 & 2.89 \\
\hline Goma Xantana & 1.44 & 1.44 & 1.44 & 1.44 \\
\hline Hexadiamina & $83.33 \mathrm{ml} / \mathrm{L}$ & $83.33 \mathrm{~mL} / \mathrm{L}$ & $83.33 \mathrm{~mL} / \mathrm{L}$ & $83.33 \mathrm{~mL} / \mathrm{L}$ \\
\hline Carbonato de Calcio & 246.16 & 246.16 & 246.16 & 246.16 \\
\hline Bentonita & 57 & 57 & 57 & 57 \\
\hline Nitrato de Potasio & 11.58 & 23.16 & 34.75 & 46.33 \\
\hline
\end{tabular}

Tabla 5. Fluido de perforación con inhibidor formiato de sodio

\begin{tabular}{|c|c|c|c|c|}
\hline & $\begin{array}{c}\text { Base } 9 \\
\mathrm{I}_{3}-\mathrm{C}_{1} \\
\end{array}$ & $\begin{array}{c}\text { Base } 10 \\
\mathrm{I}_{3}-\mathrm{C}_{2}\end{array}$ & $\begin{array}{c}\text { Base } 11 \\
\mathrm{I}_{3}-\mathrm{C}_{3}\end{array}$ & $\begin{array}{c}\text { Base } 12 \\
\mathrm{I}_{3}-\mathrm{C}_{4}\end{array}$ \\
\hline Producto & \multicolumn{4}{|c|}{ Concentración, mg/L } \\
\hline Almidón & 8.69 & 8.69 & 8.69 & 8.69 \\
\hline Carboximetil-Celulosa & 2.89 & 2.89 & 2.89 & 2.89 \\
\hline Goma Xantana & 1.44 & 1.44 & 1.44 & 1.44 \\
\hline Hexadiamina & $83.33 \mathrm{~mL} / \mathrm{L}$ & $83.33 \mathrm{~mL} / \mathrm{L}$ & $83.33 \mathrm{~mL} / \mathrm{L}$ & $83.33 \mathrm{ml} / \mathrm{L}$ \\
\hline Carbonato de Calcio & 246.16 & 246.16 & 246.16 & 246.16 \\
\hline Bentonita & 57 & 57 & 57 & 57 \\
\hline Formiato de Sodio & 11.58 & 23.16 & 34.75 & 46.33 \\
\hline
\end{tabular}

En la Tabla 6 se presentan los parámetros de control de los diferentes fluidos de perforación.

Tabla 6. Mediciones de parámetros de control en el fluido de perforación

\begin{tabular}{ccccc}
\hline Formulación base & Densidad(g/mL) & $\begin{array}{c}\text { Viscosidad } \\
\text { plástica(cps) }\end{array}$ & $\begin{array}{c}\text { Viscosidad } \\
\text { aparente(cps) }\end{array}$ & Punto cedente (cps) \\
\hline Original & 8.42 & 26 & 34 & 24 \\
1 & 8.57 & 22 & 35 & 26 \\
2 & 8.62 & 24 & 36 & 22 \\
3 & 8.55 & 24 & 35 & 24 \\
4 & 8.63 & 22 & 34 & 20 \\
5 & 8.59 & 26 & 36 & 24 \\
6 & 8.62 & 24 & 36 & 26 \\
7 & 8.67 & 22 & 35 & 26 \\
9 & 8.53 & 22 & 35 & 24 \\
10 & 8.61 & 24 & 36 & 22 \\
11 & 8.55 & 24 & 35 & 26 \\
\hline
\end{tabular}


Debido a que la adición de inhibidores químicos, como sales orgánicas e inorgánicas, influye directamente en el $\mathrm{pH}$ y la conductividad eléctrica, se realizaron medidas de estos parámetros (Tablas 7 a 9 y 10 a 12, respectivamente).

Tabla 7. Datos de los análisis químicos del pH de las muestras de suelo tratadas con la fórmula base del fluido de perforación $I_{1}$ con sus concentraciones respectivas, 10 repeticiones.

\begin{tabular}{ccccc}
\hline \multicolumn{5}{c}{$\mathbf{K}_{\mathbf{2}} \mathbf{S O}_{\mathbf{4}}$} \\
\hline Repeticiones & $\mathbf{p H}$ \\
\hline 1 & $\mathbf{C}_{\mathbf{1}}(\mathbf{1 1 . 5 8} \mathbf{g} / \mathbf{L})$ & $\mathbf{C}_{\mathbf{2}}(\mathbf{2 3 . 1 6} \mathbf{- 1} \mathbf{L})$ & $\mathbf{C}_{3}(\mathbf{3 4 . 7 5} \mathbf{g} / \mathbf{L})$ & $\mathbf{C}_{\mathbf{4}} \mathbf{( 4 6 , 3 3 \mathbf { g } / \mathbf { L } )}$ \\
\hline 2 & 3,50 & 5,70 & 7,45 & 9,50 \\
3 & 3,00 & 6,20 & 7,00 & 9,20 \\
4 & 4,25 & 6,00 & 7,50 & 8,50 \\
5 & 4,50 & 6,50 & 7,00 & 9,00 \\
6 & 4,00 & 6,50 & 7,50 & 9,50 \\
7 & 4,50 & 5,20 & 7,40 & 9,20 \\
8 & 4,50 & 5,00 & 7,25 & 8,50 \\
9 & 4,00 & 6,20 & 7,50 & 9,00 \\
10 & 4,00 & 5,00 & 7,00 & 9,50 \\
Media & 4,50 & 6,50 & 7,00 & 9,20 \\
& 4,08 & 5,88 & 7,26 & 9,11 \\
\hline
\end{tabular}

Tabla 8. Datos de los análisis químicos del pH de las muestras de suelo tratadas con la fórmula base del fluido de perforación $I_{2}$ con sus concentraciones respectivas, 10 repeticiones.

\begin{tabular}{ccccc}
\hline \multicolumn{5}{c}{$\mathbf{K N O}_{\mathbf{3}}$} \\
\hline Repeticiones & $\mathbf{C}_{\mathbf{1}}(\mathbf{1 1 . 5 8} \mathbf{\mathbf { g }} / \mathbf{L})$ & $\mathbf{C}_{\mathbf{2}}(\mathbf{2 3 . 1 6} \mathbf{g} / \mathbf{L})$ & $\mathbf{C}_{\mathbf{3}}(\mathbf{3 4 . 7 5} \mathbf{g} / \mathbf{L})$ & $\mathbf{C}_{\mathbf{4}}(\mathbf{4 6 , 3 3} \mathbf{g} \mathbf{L})$ \\
\hline 1 & 5,3 & 5,5 & 5,7 & 5,5 \\
2 & 5,5 & 5,5 & 6,0 & 5,3 \\
3 & 5,0 & 5,2 & 5,3 & 5,3 \\
4 & 5,0 & 5,3 & 5,5 & 5,5 \\
5 & 5,0 & 5,2 & 5,3 & 5,5 \\
6 & 5,5 & 5,3 & 5,5 & 5,5 \\
7 & 5,2 & 5,5 & 5,0 & 5,2 \\
8 & 5,3 & 5,5 & 5,5 & 5,0 \\
9 & 5,3 & 5,5 & 5,7 & 5,2 \\
10 & 5,2 & 5,3 & 5,5 & 5,5 \\
Media & 5,2 & 5,4 & 5,5 & 5,4 \\
\hline
\end{tabular}


Tabla 9. Datos de los análisis químicos del pH de las muestras de suelo tratadas con la fórmula base del fluido de perforación $I_{3}$ con sus concentraciones respectivas, 10 repeticiones.

\begin{tabular}{ccccc}
\hline \multicolumn{5}{c}{ NaCOOH } \\
\hline Repeticiones & $\mathbf{C}_{\mathbf{1}}(\mathbf{1 1 . 5 8 g} / \mathbf{L})$ & $\mathbf{C}_{\mathbf{2}}(\mathbf{2 3 . 1 6} \mathbf{g} / \mathbf{L})$ & $\mathbf{C}_{\mathbf{3}}(\mathbf{3 4 . 7 5} \mathbf{g} / \mathbf{L})$ & $\mathbf{C}_{\mathbf{4}}(\mathbf{4 6} \mathbf{6 3} \mathbf{3} \mathbf{g} / \mathbf{L})$ \\
\hline 1 & 8,5 & 8,7 & 9,0 & 9,2 \\
2 & 9,0 & 9,5 & 9,2 & 8,3 \\
3 & 8,5 & 9,2 & 9,5 & 9,0 \\
4 & 9,0 & 8,5 & 9,5 & 9,2 \\
5 & 8,7 & 9,2 & 8,5 & 8,5 \\
6 & 9,5 & 8,3 & 8,5 & 9,2 \\
7 & 8,2 & 8,5 & 9,2 & 8,5 \\
8 & 8,5 & 9,2 & 9,0 & 8,7 \\
9 & 8,3 & 9,5 & 8,0 & 9,2 \\
10 & 9,2 & 9,5 & 8,5 & 9,0 \\
Media & 8,7 & 9,0 & 8,9 & 8,9 \\
\hline
\end{tabular}

Tabla 10. Datos de los análisis químicos de conductividad eléctrica de las muestras de suelo tratadas con la fórmula base del fluido de perforación $I_{1}$ con sus concentraciones respectivas, 10 repeticiones.

\begin{tabular}{|c|c|c|c|c|}
\hline \multicolumn{5}{|c|}{$\mathrm{K}_{2} \mathrm{SO}_{4}$} \\
\hline \multicolumn{5}{|c|}{ C.E. $(\mathrm{mS} / \mathrm{cm})$} \\
\hline Repeticiones & $C_{1}(11.58 \mathrm{~g} / \mathrm{L})$ & $C_{2}(23.16 \mathrm{~g} / \mathrm{L})$ & $C_{3}(34.75 \mathrm{~g} / \mathrm{L})$ & $C_{4}(46,33 \mathrm{~g} / \mathrm{L})$ \\
\hline 1 & 8,0 & 8,1 & 4,7 & 20,0 \\
\hline 2 & 6,0 & 8,1 & 4,5 & 19,0 \\
\hline 3 & 8,0 & 8,2 & 4,0 & 20,0 \\
\hline 4 & 7,0 & 8,0 & 4,5 & 19,0 \\
\hline 5 & 8,0 & 8,0 & 4,5 & 19,0 \\
\hline 6 & 7,0 & 8,0 & 4,3 & 19,0 \\
\hline 7 & 8,0 & 8,5 & 4,7 & 19,0 \\
\hline 8 & 7,0 & 8,0 & 4,5 & 20,0 \\
\hline 9 & 8,0 & 8,5 & 4,5 & 20,0 \\
\hline 10 & 7,0 & 8,2 & 4,7 & 20,0 \\
\hline Media & 7,4 & 8,2 & 4,5 & 19,5 \\
\hline
\end{tabular}


Tabla 11. Datos de los análisis químicos de conductividad eléctrica de las muestras de suelo tratadas con la fórmula base del fluido de perforación $I_{2}$ con sus concentraciones respectivas, 10 repeticiones.

\begin{tabular}{|c|c|c|c|c|}
\hline \multicolumn{5}{|c|}{$\mathrm{KNO}_{3}$} \\
\hline \multicolumn{5}{|c|}{ C.E. (mS/cm) } \\
\hline Repeticiones & $C_{1}(11.58 \mathrm{~g} / L)$ & $C_{2}(23.16 g / L)$ & $C_{3}(34.75 g / L)$ & $C_{4}(46,33 \mathrm{~g} / \mathrm{L})$ \\
\hline 1 & 3,5 & 3,5 & 3,7 & 3,5 \\
\hline 2 & 3,7 & 4,3 & 3,5 & 3,7 \\
\hline 3 & 4,0 & 4,0 & 3,7 & 4,0 \\
\hline 4 & 3,5 & 4,5 & 3,5 & 3,5 \\
\hline 5 & 4,2 & 3,5 & 3,5 & 2,5 \\
\hline 6 & 3,5 & 4,3 & 4,0 & 3,7 \\
\hline 7 & 3,7 & 3,7 & 4,4 & 3,5 \\
\hline 8 & 4,2 & 3,5 & 3,5 & 3,7 \\
\hline 9 & 4,4 & 3,5 & 3,7 & 3,5 \\
\hline 10 & 3,7 & 4,0 & 3,7 & 3,5 \\
\hline Media & 3,8 & 3,9 & 3,7 & 3,5 \\
\hline
\end{tabular}

Tabla 12. Datos de los análisis químicos de conductividad eléctrica de las muestras de suelo tratadas con la fórmula base del fluido de perforación $I_{3}$ con sus concentraciones respectivas, 10 repeticiones.

\section{NaCOOH}

\section{C.E. (mS/cm)}

\begin{tabular}{ccccc}
\hline Repeticiones & $\mathbf{C}_{\mathbf{1}}(\mathbf{1 1 . 5 8 g} / \mathbf{L})$ & $\mathbf{C}_{\mathbf{2}}(\mathbf{2 3 . 1 6} \mathbf{g} / \mathbf{L})$ & $\mathbf{C}_{\mathbf{3}}(\mathbf{3 4 . 7 5} \mathbf{g} / \mathbf{L})$ & $\mathbf{C}_{\mathbf{4}}(\mathbf{4 6}, \mathbf{3 3} \mathbf{g} / \mathbf{L})$ \\
\hline 1 & 66,7 & 65,5 & 67,2 & 64,5 \\
2 & 65,3 & 66,7 & 66,7 & 65,7 \\
3 & 68,5 & 64,5 & 65,5 & 66,0 \\
4 & 65,4 & 68,0 & 64,3 & 65,3 \\
5 & 66,8 & 65,2 & 66,5 & 64,8 \\
6 & 60,5 & 66,5 & 64,3 & 62,7 \\
7 & 65,4 & 64,8 & 65,5 & 65,5 \\
8 & 63,5 & 66,7 & 66,7 & 66,5 \\
9 & 66,5 & 65,4 & 66,2 & 65,7 \\
10 & 66,7 & 63,2 & 64,7 & 66,4 \\
Media & 66,7 & 65,7 & 65,8 & 65,3 \\
\hline
\end{tabular}




\subsection{Interpretación de resultados}

En las formulaciones base del fluido de perforación (Tablas 3 hasta 5), se determinó la densidad y viscosidad plástica, datos planteados en la Tabla 6, y se calculó la viscosidad aparente y el punto cedente de cada una de ellas. Mediante los cuales podemos decir que las formulaciones de los fluidos cumplen con las propiedades reológicas adecuadas para el proceso de perforación, donde los valores entran en el rango establecido en el manual de fluidos de perforación del Instituto Americano de Petróleo (API), viscosidad plástica (20-30 cps), viscosidad aparente (>30 cps) y punto cedente (>15 cps).
El valor de la viscosidad plástica en la formulación base 5 (Tabla 4), es $26 \mathrm{cps}$, significa que el fluido de perforación es más viscoso y por ende necesita más energía durante el bombeo en el proceso de perforación.

Los resultados obtenidos de $\mathrm{pH}$ en las muestras de suelo (Tablas 7 hasta 9), y conductividad eléctrica (Tablas 10 hasta 12), fueron evaluados mediante el análisis de varianza de dos factores con una sola muestra, la probabilidad indica a qué nivel los resultados son estadísticamente significativos o no significativos (Tabla 13).

Tabla 13. Análisis de varianza del pH en las muestras de suelo con $I_{1}$.

\begin{tabular}{|c|c|c|c|c|c|}
\hline $\mathrm{pH} \mathrm{K} \mathrm{K}_{2} \mathrm{SO}_{4}$ & $\begin{array}{l}\text { Suma de } \\
\text { cuadrados }\end{array}$ & Grados de libertad & $\begin{array}{l}\text { Promedio de } \\
\text { los cuadrados }\end{array}$ & $\mathbf{F}$ & $\begin{array}{l}\text { Valor } \\
\text { crítico } \\
\text { para F }\end{array}$ \\
\hline Repeticiones & 1,76 & 9,00 & 0,20 & 1,04 & 2,46 \\
\hline Concentraciones & 52,53 & 2,00 & 26,27 & 139,03 & 3,55 \\
\hline Total & 57,70 & 29,00 & & & \\
\hline
\end{tabular}

En la Tabla 13, por medio del análisis de varianza, con un nivel de confianza del $95 \%$ se puede evidenciar claramente que no hay diferencia significativa en sus repeticiones $(1,04<2,46)$, en cambio al analizar las concentraciones indica que sí hay diferencia significativa $(139>5,55)$, entre las medidas del $\mathrm{pH}$ con las concentraciones aplicadas. El suelo tratado obtuvo un
$\mathrm{pH}$ de 7.26, valor medio que está dentro del límite requerido por la ley hidrocarburifera, ver Tabla 7 y fue con la concentración de $34.75 \mathrm{~g} / \mathrm{L}$, del inhibidor $\mathrm{K}_{2} \mathrm{SO}_{4}$.

La Tabla 14 presenta el análisis de varianza para la conductividad eléctrica.

Tabla 14 Análisis de varianza de la conductividad eléctrica en las muestras de suelo con $I_{1}$.

\begin{tabular}{cccccc}
\hline C.E. $\mathbf{K}_{\mathbf{2}} \mathbf{S O}_{\mathbf{4}}$ & $\begin{array}{c}\text { Suma de } \\
\text { cuadrados }\end{array}$ & $\begin{array}{c}\text { Grados de } \\
\text { libertad }\end{array}$ & $\begin{array}{c}\text { Promedio de } \\
\text { los cuadrados }\end{array}$ & F & $\begin{array}{c}\text { Valor crítico } \\
\text { para F }\end{array}$ \\
\hline Repeticiones & 2,78 & 9,00 & 0,31 & 1,71 & 2,25 \\
Concentraciones & 1307,05 & 3,00 & 435,68 & 2412,15 & 2,96 \\
Total & 1314,70 & 39,00 & & & \\
\hline
\end{tabular}

En la tabla 14, por medio del análisis de varianza, con un nivel de confianza del $95 \%$ se puede evidenciar claramente que no hay diferencia significativa en sus repeticiones $(1,71<2,25)$, en cambio al analizar las concentraciones indica que sí hay diferencia significativa entre las medidas de la conductividad eléctrica $(2412>2,96)$, con las concentraciones aplicadas. El suelo tratado obtuvo un valor de $4.49 \mathrm{mS} / \mathrm{cm}$, valor medio que está dentro del límite requerido por la ley hidrocarburifera, ver Tabla 10 y fue con la concentración de $34.75 \mathrm{~g} / \mathrm{L}$, del inhibidor $\mathrm{K}_{2} \mathrm{SO}_{4}$.

El crecimiento de la planta de fréjol se puede evidenciar en la parcela que contiene $\mathrm{K}_{2} \mathrm{SO}_{4}$, a una concentración de $34,75 \mathrm{~g} / \mathrm{L}$. A una concentración baja el inhibidor deshidrata a la planta, afectando algunas etapas de su crecimiento y a concentraciones altas la semilla se satura y no permite su germinación (Figura 1). 


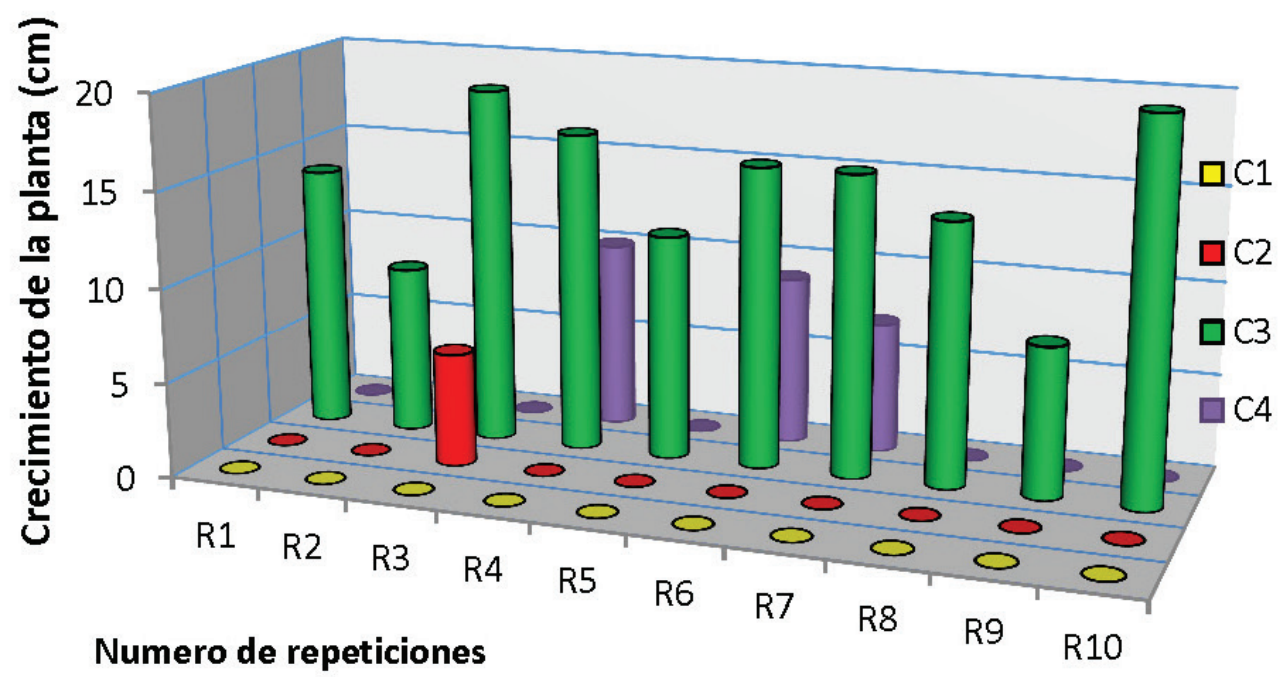

Figura 1. Esquema del crecimiento de la planta de fréjol con $\mathrm{K}_{2} \mathrm{SO}_{4}$, con sus concentraciones respectivas y 10 repeticiones.

Para el inhibidor nitrato de potasio se realizó el análisis de varianza para el pH (Tabla 15) y conductividad (Tabla 16).

Tabla 15. Análisis de varianza del pH en las muestras de suelo con $I_{2}$

\begin{tabular}{cccccc}
\hline pH KNO & $\begin{array}{c}\text { Suma de } \\
\text { cuadrados }\end{array}$ & $\begin{array}{c}\text { Grados de } \\
\text { libertad }\end{array}$ & $\begin{array}{c}\text { Promedio de } \\
\text { los cuadrados }\end{array}$ & F & $\begin{array}{c}\text { Valor crítico } \\
\text { para F }\end{array}$ \\
\hline Repeticiones & 0,55 & 9,00 & 0,06 & 1,87 & 2,25 \\
Concentraciones & 0,37 & 3,00 & 0,12 & 2,79 & 2,96 \\
Total & 1,79 & 39,00 & & & \\
\hline
\end{tabular}

En la Tabla 15, por medio del análisis de varianza, nes de igual forma $(2,79<2,96)$. El suelo tratado obcon un nivel de confianza del $95 \%$ se puede eviden- tuvo un $\mathrm{pH}$ de entre (5-6), valores que no cumplen el ciar claramente que no hay diferencia significativa en límite permisible de acuerdo a la ley hidrocarburifera, sus repeticiones $(1,87<2,25)$ ni en sus concentracio- ver Tabla 8 .

Tabla 16. Análisis de varianza de la conductividad eléctrica en las muestras de suelo con $I_{2}$.

\begin{tabular}{lccccc}
\hline \multicolumn{1}{c}{ C.E. KNO } & $\begin{array}{c}\text { Suma de } \\
\text { cuadrados }\end{array}$ & Grados de libertad & $\begin{array}{c}\text { Promedio de } \\
\text { los cuadrados }\end{array}$ & F & $\begin{array}{c}\text { Valor crítico } \\
\text { para F }\end{array}$ \\
\hline Repeticiones & 0,80 & 9,00 & 0,09 & 0,65 & 2,25 \\
Concentraciones & 0,83 & 3,00 & 0,28 & 2,02 & 2,96 \\
Total & 5,31 & 39,00 & & & \\
\hline
\end{tabular}

En la Tabla 16, por medio del análisis de varianza, con un nivel de confianza del $95 \%$ se puede evidenciar claramente que no hay diferencia significativa en sus repeticiones $(0,6<2,25)$ ni en sus concentraciones de igual forma $(2,02<2,96)$. El suelo tratado obtuvo un valor de conductividad eléctrica entre $(3-4 \mathrm{mS} / \mathrm{cm})$, valores que no cumplen el límite permisible de acuerdo a la ley hidrocarburífera, ver Tabla 11 . 
En las parcelas donde se puso el fluido de perforación con el inhibidor de nitrato de potasio $\mathrm{KNO}_{3}$ crecieron 4 plantas de las 10 semillas repartidas y a la concentración de $11.58 \mathrm{~g} / \mathrm{L}$, todo esto es debido a la saturación de agua que hay en el suelo por parte del fluido de perforación y la aportación de nitrógeno en forma nítrica que afecta algunas etapas de su crecimiento, evidentemente a mas concentración de nitratos la inhibición o toma física de agua hace que se vuelva más tóxico el suelo, perturbando su desarrollo a manera que la concentración del inhibidor vaya aumentando (Figura 2).

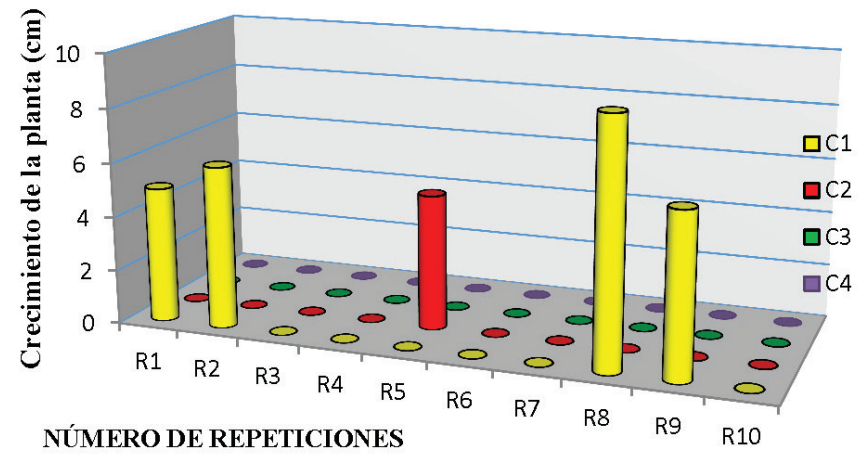

Figura 2. Esquema del crecimiento de la planta de frejol con $\mathrm{KNO}_{3^{\prime}}$ con sus concentraciones respectivas y 10 repeticiones.

Para el inhibidor formiato de sodio se realizó el análisis de varianza para el pH (Tabla 17) y conductividad (Tabla 18).

Tabla 17. Análisis de varianza del pH en las muestras de suelo con $I_{3}$.

\begin{tabular}{cccccc}
\hline pH NacoOH & $\begin{array}{c}\text { Suma de } \\
\text { cuadrados }\end{array}$ & $\begin{array}{c}\text { Grados de } \\
\text { libertad }\end{array}$ & $\begin{array}{c}\text { Promedio de } \\
\text { los cuadrados }\end{array}$ & F & $\begin{array}{c}\text { Valor crítico } \\
\text { para F }\end{array}$ \\
\hline Repeticiones & 0,88 & 9,00 & 0,10 & 0,43 & 2,25 \\
Concentraciones & 0,35 & 3,00 & 0,12 & 0,52 & 2,96 \\
Total & 7,32 & 39,00 & & & \\
\hline
\end{tabular}

En la Tabla 17, por medio del análisis de varianza, con un nivel de confianza del $95 \%$ se puede evidenciar claramente que no hay diferencia significativa en sus repeticiones $(0,43<2,25)$ ni en sus concentracio- nes de igual forma $(0,52<2,96)$. El suelo tratado obtuvo un $\mathrm{pH}$ entre $(8-9,5)$, valores que no cumplen el limite permisible de acuerdo a la ley hidrocarburifera, ver Tabla 9.

Tabla 18. Análisis de varianza de la conductividad eléctrica en las muestras de suelo con $I_{3}$.

\begin{tabular}{ccccccc}
\hline C.E. NacoOH & $\begin{array}{c}\text { Suma de } \\
\text { cuadrados }\end{array}$ & $\begin{array}{c}\text { Grados de } \\
\text { libertad }\end{array}$ & $\begin{array}{c}\text { Promedio de los } \\
\text { cuadrados }\end{array}$ & F & $\begin{array}{c}\text { Valor } \\
\text { crítico } \\
\text { para F }\end{array}$ \\
\hline Repeticiones & 22,15 & 9,00 & 2,16 & 1,12 & 2,25 \\
Concentraciones & 1,11 & 3,00 & 0,37 & 0,17 & 2,96 \\
Total & 82,76 & 39,00 & & & \\
\hline
\end{tabular}


En la Tabla 18, por medio del análisis de varianza, con un nivel de confianza del $95 \%$ se puede evidenciar claramente que no hay diferencia significativa en sus repeticiones $(1,12<2,25)$ ni en sus concentraciones de igual forma $(0,17<2,96)$. El suelo tratado obtuvo un valor de conductividad eléctrica $160-70 \mathrm{mS} /$ $\mathrm{cm})$, valores que no cumplen el limite permisible de acuerdo a la ley hidrocarburífera, ver Tabla 12 .

Las parcelas en donde se puso el fluido de perforación con el inhibidor de formiato de sodio, $\mathrm{NaCOOH}$, se observa un deterioro completo de la semilla, su pH está entre (8-10) y su conductividad entre (50-70 $\mu \mathrm{S} /$ $\mathrm{cm})$, parámetros que tampoco entran en el rango permisible de la ley hidrocarburífera, todo esto es debido a que el suelo se volvió totalmente salino provocando un crecimiento desfavorable de la semilla, pues se dice que en la semilla se forma una película hidrofóbica a su alrededor impidiendo la entrada de agua provocando estrés hídrico afectando su germinación (Figura 3).

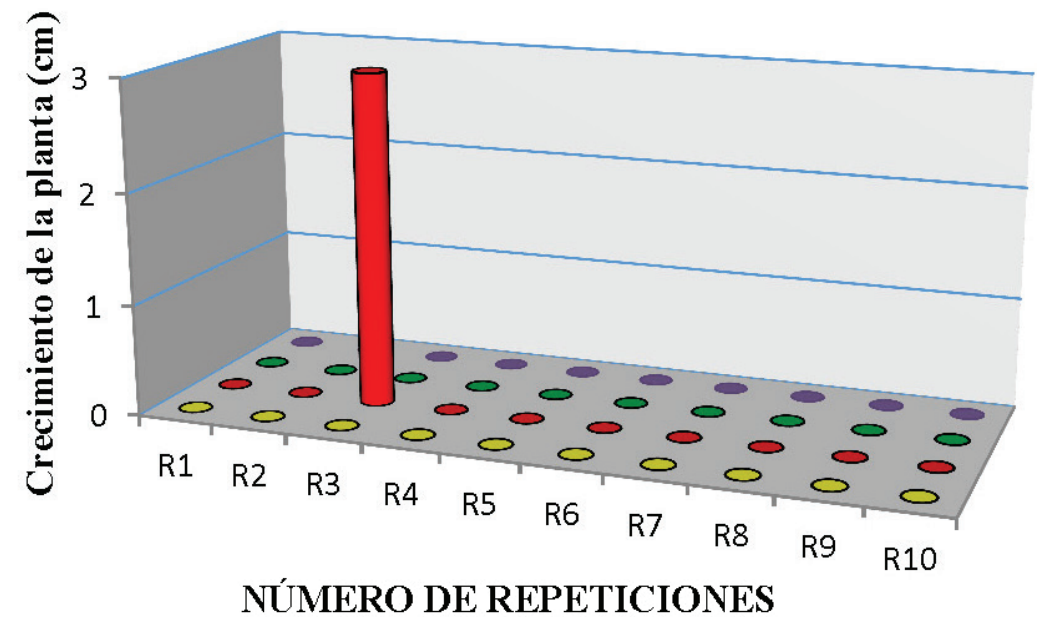

Figura 3. Esquema del crecimiento de la planta de fréjol con $\mathrm{NaCOOH}$ con sus concentraciones respectivas y 10 repeticiones.

\section{Conclusiones}

- Al diseñar un fluido de perforación en base a los métodos del Instituto Americano del Petróleo (API), utilizando productos químicos como: almidón carboximetil celulosa, goma xantana, carbonato de calcio, bentonita como fórmula base y adicionando como inhibidores: $\mathrm{K}_{2} \mathrm{SO}_{4}, \mathrm{KNO}_{3}$ y $\mathrm{NaCOOH}$, a diferentes concentraciones: $11,58 \mathrm{~g} / \mathrm{L}-23,16 \mathrm{~g} / \mathrm{L}-34,75$ $\mathrm{g} / \mathrm{L}-46,33 \mathrm{~g} / \mathrm{L}$, se aprecia que las propiedades reológicas medidas como parámetros de control estuvieron dentro de los rangos establecidos en la metodología, 20-30 cps de viscosidad plástica, $>30 \mathrm{cps}$ de viscosidad aparente, $>15 \mathrm{cps}$ de punto cedente y 8-9 $\mathrm{g} / \mathrm{mL}$ de densidad; por lo tanto, se concluye que los inhibidores utilizados en la presente investigación no influyeron en la propiedades reológicas.
- El mejor fluido de perforación que en su disposición final no afecta el suelo fue el que contiene sulfato de potasio a una concentración de $34,75 \mathrm{~g} / \mathrm{L}$, ya que se obtuvieron valores promedio de $\mathrm{pH} \mathrm{7,26}$ y conductividad eléctrica 4,49 mS/cm, que están dentro del límite requerido por la Ley Hidrocarburífera.

- Los mejores resultados en el crecimiento de la planta de fréjol se dieron en la parcela \#3, al mezclar el fluido de perforación que contiene: almidón 8,69 g/L, carboximetil celulosa 2,89 $\mathrm{g} / \mathrm{L}$, goma xantana $1,44 \mathrm{~g} / \mathrm{L}$, hexadiamina $83.33 \mathrm{~mL} / \mathrm{L}$, carbonato de calcio 246,16 $\mathrm{g} / \mathrm{L}$, bentonita $57 \mathrm{~g} / \mathrm{L}$, y sulfato de potasio $34,75 \mathrm{~g} / \mathrm{L}$ con el suelo, según se evidenció cuando las diez plantas de fréjol crecieron 14 $\mathrm{cm}$, en promedio, en esta parcela. 


\section{Recomendaciones}

- Para la elaboración del fluido de perforación es necesario seguir el procedimiento basado en el manual de métodos API de cada empresa prestadora de servicios petroleros, ya que los productos químicos utilizados durante el proceso pueden sufrir alguna descomposición y por la cantidad que se utiliza no puede arriesgarse a desperdiciar producto.

- El período de 180 días que establece la Ley Hidrocarburífera (Decreto 1215) para el análisis de suelo después de la disposición final de los fluidos de perforación es importante, ya que después de este tiempo los resultados de los análisis químicos indican si el suelo necesita biorremediación o si su contaminación disminuyo a un porcentaje adecuado para reutilización del mismo.

- Es importante recomendar el uso de plantas de las que se conozca previamente la cantidad de brote, condiciones de desarrollo y tiempo de vida, como en el caso de la planta de fréjol, utilizada en la presente investigación.

\section{Referencias}

Fernández, L., Rojas, G., Roldán, T., \& Ramirez, E. (2006). Manual de técnicas de análisis de suelos aplicadas a la remediación de sitios contaminados. México: INE, IMP.

Instituto Americano del Petróleo. (2001). Procedimiento Estándar para las Pruebas de Fluidos de Perforación. Dallas: API.

Ministerio del Ambiente, (2012): Línea Base de Deforestación del Ecuador Continental, MAE: Quito-Ecuador

Petroproducción. (1992). UNIDAD DE MANEJO AMBIENTAL: Manejo de desechos: sólidos, líquidos y gaseosos. Quito: Petroproducción.

SENPLADES (2009). República del Ecuador, Plan Nacional de Desarrollo. Plan Nacional del Buen Vivir 2009 - 2013: Construyendo un Estado

Plurinacional e Intercultural. Quito: Secretaría Nacional de Planificación y Desarrollo 